\title{
Bacterial Viruses Subcommittee and Archaeal Viruses Subcommittee of the ICTV: update of taxonomy changes in 2021
}

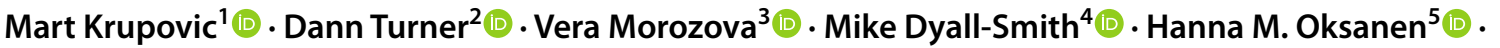

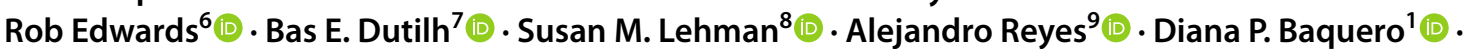 \\ Matthew B. Sullivan ${ }^{10,11}$ (1) J Jumpei Uchiyama ${ }^{12}$ (D) Jesca Nakavuma ${ }^{13}$ (D) Jakub Barylski ${ }^{14} \cdot$ Mark J. Young $^{15}$ (0) \\ Shishen $\mathrm{Du}^{16}$. Poliane Alfenas-Zerbini ${ }^{17(\mathbb{0})}$ - Alla Kushkina ${ }^{18}$ (1) Andrew M. Kropinski ${ }^{19,20}$.

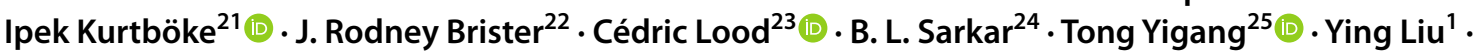

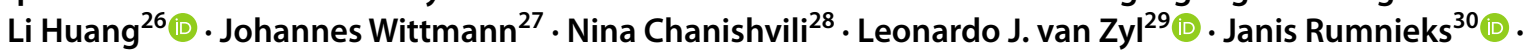 \\ Tomohiro Mochizuki ${ }^{31}$ (1) Matti Jalasvuori ${ }^{32}$ (D) Ramy K. Aziz ${ }^{33}$ (1) Małgorzata Łobocka ${ }^{34}$ (D) \\ Kenneth M. Stedman ${ }^{35}$ (D) Andrey N. Shkoporov ${ }^{36}$ - Annika Gillis ${ }^{37}$ (D) Xu Peng ${ }^{38}$ (D) François Enault ${ }^{39}$ (i)

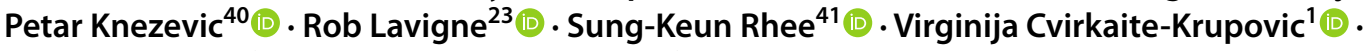 \\ Cristina Moraru ${ }^{42}$ - Andrea I. Moreno Switt ${ }^{43}$ - Minna M. Poranen ${ }^{5} \cdot$ Andrew Millard $^{44}\left(\right.$ David Prangishvili $^{45}$. \\ Evelien M. Adriaenssens ${ }^{46}{ }^{(1)}$
}

Published online: 21 August 2021

(c) The Author(s) 2021

\begin{abstract}
In this article, we - the Bacterial Viruses Subcommittee and the Archaeal Viruses Subcommittee of the International Committee on Taxonomy of Viruses (ICTV) - summarise the results of our activities for the period March 2020 - March 2021. We report the division of the former Bacterial and Archaeal Viruses Subcommittee in two separate Subcommittees, welcome new members, a new Subcommittee Chair and Vice Chair, and give an overview of the new taxa that were proposed in 2020, approved by the Executive Committee and ratified by vote in 2021. In particular, a new realm, three orders, 15 families, 31 subfamilies, 734 genera and 1845 species were newly created or redefined (moved/promoted).
\end{abstract}

\section{Changes in Subcommittee structure and membership}

Bacteriophage and archaeal virus taxonomy has been formally under the auspices of the ICTV Bacterial and Archaeal Viruses Subcommittee, which, at its inception in 1966, was named the Viruses of Prokaryotes Subcommittee, led by David E. Bradley (https://talk.ictvonline.org/information/ w/ictv-history). Given the revived interest in bacterial and archaeal viruses and recent enormous increase in the number of characterized isolates and need for creation of numerous taxa to classify them, the Executive Committee (EC) voted on the creation of two separate Subcommittees (EC51, July 2019), formally starting their mandates after EC52 (October

Handling Editor: Sead Sabanadzovic.

Evelien M. Adriaenssens

evelien.adriaenssens@quadram.ac.uk

Extended author information available on the last page of the article
2020). The new Bacterial Viruses Subcommittee is chaired by Evelien Adriaenssens, supported by Dann Turner as the Vice Chair, and the new Archaeal Viruses Subcommittee is chaired by Mart Krupovic. Both Chairs were elected for three-year terms ending in 2023. As a result, this taxonomy update summarises both bacterial and archaeal virus proposals for the last time, reflecting the fact that proposals were submitted prior to the formal reorganisation of the original Subcommittee.

In the new Bacterial Viruses Subcommittee, we continue our structure of Study Groups (SGs), regional representatives and general members. We would like to welcome new members Jesca Nakavuma (Uganda), Alejandro Reyes (Colombia), Cristina Moraru (Germany), Susan Lehman (USA), Cédric Lood (Belgium) and Andrey Shkoporov (Ireland) and would like to thank those who have left the Subcommittee for their service.

In the framework of the Bacterial and Archaeal Viruses Subcommittee, all taxonomic proposals dealing with archaeal viruses were handled by a single SG. The new 
Archaeal Viruses Subcommittee currently includes 11 SGs (Table 1), each created for a three-year term. Of note, the Halopanivirales SG, chaired by Hanna M. Oksanen (Finland), oversees the taxonomy of evolutionarily related viruses of the order Halopanivirales, which includes two families of viruses infecting archaea (Sphaerolipoviridae and Simuloviridae) and one family of bacteriophages (Matsushitaviridae). Thus, the latter SG bridges the two Subcommittees. Additional SGs will be created in the near future, in particular, to develop the taxonomy of archaeal members of the class Caudoviricetes; only a handful of isolated representatives of this highly diverse and expansive group of archaeal viruses are currently classified.

\section{Taxonomy update}

We had a record year of submissions of taxonomy proposals in 2020, with 188 proposals submitted, of which all but one were approved and ratified (Supplementary Table S1). The changes at each of the taxonomic ranks in use for bacterial and archaeal viruses are summarised in Table 2. We
Table 1 Composition of the archaeal viruses subcommittee

\begin{tabular}{|c|c|c|}
\hline Study Group & Member & Country \\
\hline \multirow[t]{4}{*}{ Bicaudaviridae SG } & Mart Krupovic* & France \\
\hline & Li Huang & China \\
\hline & Mark J. Young & USA \\
\hline & Virginija Cvirkaite-Krupovic & France \\
\hline \multirow[t]{2}{*}{ Desulfurococcales viruses SG } & Tomohiro Mochizuki* & Japan \\
\hline & Mart Krupovic & France \\
\hline \multirow[t]{2}{*}{ Fuselloviridae SG } & Kenneth M. Stedman* & USA \\
\hline & Mart Krupovic & France \\
\hline \multirow[t]{4}{*}{ Halopanivirales SG } & Hanna M. Oksanen* & Finland \\
\hline & Mike Dyall-Smith & Australia \\
\hline & Shishen Du & China \\
\hline & Matti Jalasvuori & Finland \\
\hline \multirow[t]{2}{*}{ Halspiviridae SG } & Mike Dyall-Smith* & Australia \\
\hline & Hanna M. Oksanen & Finland \\
\hline Ovaliviridae SG & Li Huang* & China \\
\hline \multirow[t]{3}{*}{ Pleolipoviridae SG } & Hanna M. Oksanen* & Finland \\
\hline & Mike Dyall-Smith & Australia \\
\hline & Ying Liu & France \\
\hline \multirow[t]{2}{*}{ Portogloboviridae SG } & Mart Krupovic* & France \\
\hline & Ying Liu & France \\
\hline \multirow[t]{2}{*}{ Thaspiviridae SG } & Sung-Keun Rhee* & Republic of Korea \\
\hline & Mart Krupovic & France \\
\hline \multirow[t]{4}{*}{ Tokiviricetes SG } & Mart Krupovic* & France \\
\hline & Tomohiro Mochizuki & Japan \\
\hline & Xu Peng & Denmark \\
\hline & Diana P. Baquero & France \\
\hline Turriviridae SG & Mark J. Young* & USA \\
\hline
\end{tabular}

*Study Group Chair

\begin{tabular}{llllllllll}
\hline & Species & Genus & Subfamily & Family & Order & Class & Phylum & Kingdom & Realm \\
\hline Abolished & 20 & 2 & 0 & 0 & 0 & 0 & 0 & 0 & 0 \\
New & 1775 & 700 & 28 & 14 & 2 & 1 & 1 & 1 & 1 \\
$\begin{array}{l}\text { Moved or pro- } \\
\text { moted (and/or }\end{array}$ & 70 & 34 & 3 & 1 & 1 & 0 & 0 & 0 & 0 \\
$\begin{array}{r}\text { renamed) } \\
\text { Renamed }\end{array}$ & 33 & 8 & 0 & 1 & 1 & 1 & 0 & 0 & 0 \\
\hline
\end{tabular}

Table 2 Summary of taxonomic changes for bacterial and archaeal viruses for Master Species List 36, ratified March 2021 
created a record 1775 new species, 700 new genera, 28 new subfamilies, 14 new families and two new orders and also created one new realm containing one new kingdom, one new phylum and one new class. Given the large numbers of proposals and taxa that were created, moved or renamed, it becomes unfeasible to describe all the changes in detail; however, we urge interested readers to consult Supplementary Table S1 and the associated proposals from the ICTV website (https://talk.ictvonline.org/files/ictv_official_taxon omy_updates_since_the_8th_report/m/prokaryote-official). Instead, below we provide a brief overview of the most notable changes.

\section{The new realm Adnaviria, a new megataxonomy of archaeal filamentous viruses}

Recently, the taxonomic framework of the ICTV has been expanded from five to 15 ranks, with the highest-level rank, realm, being equivalent to the domain rank used for cellular organisms [1]. Until recently, four such realms had been established for classification of viruses infecting hosts from different domains of life [2,3]. This year, a new realm, Adnaviria, was created for classification of archaeal filamentous viruses with dsDNA genomes that adopt the A-form conformation within their virions $[4,5]$. The realm includes three families, Tristromaviridae, Rudiviridae and Lipothrixviridae, which are evolutionarily related to each other, but not to any other known group of viruses. The families Rudiviridae and Lipothrixviridae were already grouped together in the order Ligamenvirales [6], and a single-family order, Primavirales, has now been created for the family Tristromaviridae. The two orders are unified in the class Tokiviricetes, kingdom Zilligvirae and realm Adnaviria.

\section{Class Caudoviricetes, order Caudovirales}

We delineated three new families of short-tailed phages with small genomes that were formerly assigned to the family Podoviridae. The family Salasmaviridae, named in honour of Margarita Salas Falgueras, comprises the existing subfamily Picovirinae, which includes the classical bacillus phage $\varphi 29$ and a range of related Bacillus-infecting phages with genomes between 18 and $27 \mathrm{~kb}$ in size. The family Rountreeviridae, named in honour of Phyllis Margaret Rountree, groups Enterococcus-infecting phages with genomes between 17 and $19 \mathrm{~kb}$ in size, whereas the family Guelinviridae, named after Antonina Guelin, groups Clostridiuminfecting phages with genome sizes between 16 and $19 \mathrm{~kb}$.

The new family Schitoviridae, named after Giancarlo Schito, is the formalisation of the group of N4-like phages defined by the presence of a large virion-associated RNA polymerase, described in more detail in reference [7].

The new family Zobellviridae, named after Claude Zobell, groups a set of globally distributed podoviruses associated with marine ecosystems first proposed by Bischoff and colleagues [8].

\section{Class Leviviricetes}

Based on the investigation by Callanan and colleagues on the expansion of known ssRNA virus genomes [9], the family Leviviridae was elevated to the rank of class, named Leviviricetes (replacing the class Allassoviricetes), and expanded to include two orders (Norzivirales and Timlovirales) and six new families: Fiersviridae (renamed from the original family Leviviridae), Atkinsviridae, Duinviridae, Solspiviridae, Blumeviridae and Steitzviridae. A detailed description of the new taxa will be published separately.

\section{Class Tectiliviricetes, new family Autolykiviridae}

The new family Autolykiviridae formalises the group of nontailed dsDNA bacteriophages discovered and described by Kauffmann and colleagues [10], combining features of both corticoviruses and tectiviruses. This new family contains two new genera and five new species.

\section{Order Halopanivirales, new families Matsushitaviridae and Simuloviridae}

Until recently, the family Sphaerolipoviridae, which includes icosahedral tailless viruses with internal membranes, consisted of three genera, Alphasphaerolipovirus, Betasphaerolipovirus and Gammasphaerolipovirus. The first two of these genera included viruses infecting halophilic archaea, whereas the last one included phages infecting thermophilic bacteria [11]. Although viruses from the three genera are evolutionarily related [12], they display considerable sequence divergence. Thus, the genera Betasphaerolipovirus and Gammasphaerolipovirus have been renamed and moved from the Sphaerolipoviridae into new families, Simuloviridae and Matsushitaviridae (named after Isao Matsushita), respectively. The order Halopanivirales now contains a family of bacterial viruses, Matsushitaviridae, and two families of archaeal viruses, family Sphaerolipoviridae and Simuloviridae. As mentioned above, the order is under purview of a single Study Group, which is part of both the Archaeal Viruses SC and the Bacterial Viruses SC. 


\section{Order Tubulavirales, new family Paulinoviridae}

The new family Paulinoviridae addresses the challenge of defining family demarcation criteria for phages with small genomes, where members of the same family would share at least two orthologous proteins. Informed by prior work on a gene-content network of predicted filamentous prophage sequences [13], we moved the genera Bifilivirus and Thomixvirus from the family Inoviridae to the family Paulinoviridae.

\section{Online (10th) Report of the ICTV}

Virus Taxonomy: The Classification and Nomenclature of Viruses - The Online (10th) Report of the ICTV is freely accessible at http://ictv.global/report, and summaries of the chapters on each virus family are published in the Journal of General Virology. In 2020, four new chapters on bacterial and archaeal viruses were produced by members of the Archaeal Viruses SC and Bacterial Viruses SC, namely, on the families Herelleviridae [14], Spiraviridae [15], Ovaliviridae [16] and Finnlakeviridae [17].

\section{Conclusion}

This past year has been extremely productive in terms of new bacterial and archaeal virus taxa described. It would not have been possible without an active pool of scientists, both within and outside the subcommittee and its study groups, who scour databases, perform analyses and submit proposals. We continue to encourage people to contact us to formalise new discoveries into the taxonomic framework and will keep reaching out to interested parties. Finally, we would like to acknowledge one person in particular, Prof Andrew Kropinski, the Subcommittee Chair from 2014-2020, who authored, co-authored and/or assisted with the majority of proposals that have been approved in the last decade.

Supplementary Information The online version contains supplementary material available at https://doi.org/10.1007/s00705-021-05205-9.

Acknowledgements We would like to acknowledge the invaluable help from colleagues at NCBI, in particular Igor Tolstoy.

Author contributions All authors contributed to the creation or assessment of taxonomy proposals; M.K. and E.M.A wrote the manuscript with input from D.T. and A.M.K; all authors evaluated and approved the final version.

Funding V.M. was supported by Russian Ministry of Education and Science Project No. 0245-2021-0008; H.M.O. was supported by University of Helsinki funding for FINStruct and Instruct-ERIC research infrastructure; R.A.E. was supported by the National Institute Of Diabetes And Digestive And Kidney Diseases of the National Institutes of Health under Award Number RC2DK116713; B.E.D. was supported by the European Research Council (ERC) Consolidator grant 865694: DiversiPHI; M.B.S. was supported by National Science Foundation Advances in Biological Infrastructure award \#1758974; M.Y. acknowledges funding by the Gordon and Betty Moore Foundation grant GBMF9195; S.D. was supported by National Natural Science Foundation of of China grant 32070032; C.L. is supported by the Fonds Wetenschappelijk Onderzoek (1S64718N); L.H. was funded by the Strategic Priority Research Program of the Chinese Academy of Sciences Award XDB42000000,National Natural Science Foundation of China (NSFC) grant 31970170; T.M. was supported by JSPS KAKENHI Grant Numbers 21H02100, 19H04827, 18K14372, and JST JPMJJR2005, and SUMITOMO Fund 200673; MJ is supported by Academy of Finland grants \#336518 and \#297049 and Jane and Aatos Erkko Foundation; R.K.A. is supported by the Egyptian Academy of Scientific Research and Technology (ASRT) project \#3046 (JESOR); M.L. is supported by the statutory funds for the Institute of Biochemistry and Biophysics of the Polish Academy of Sciences; K.M.S. is supported by the US. National Science Foundation (MCB1929273 and MCB2025305); A.N.S. is supported by Wellcome Trust Research Career Development Fellowship (220646/Z/20/Z) and by European Research Council (ERC) grant under the European Union's Horizon 2020 research and innovation programme (grant agreement 101001684); X.P. is supported by Novo Nordisk Foundation/Hallas-Møller Ascending Investigator Grant (grant number NNF17OC0031154) and the Danish Council for Independent Research/ FNU (grant number DFF-0135-00402); S.K.R. was supported by the National Research Foundation of Korea (NRF) grants funded by the Ministry of Science, ICT \& Future Planning (2021R1A2C3004015); C.M. acknowledges funding by the Deutsche Forschungsgemeinschaft within the Collaborative Research Center TRR51 Roseobacter (INST 184/170-1); M.M.P. acknowledges funding from the Academy of Finland (grant 331627) and the Sigrid Juselius Foundation; E.M.A. gratefully acknowledges funding by the Biotechnology and Biological Sciences Research Council (BBSRC); this research was funded by the BBSRC Institute Strategic Programme Gut Microbes and Health BB/R012490/1 and its constituent projects BBS/E/F/000PR10353 and BBS/E/F/000PR10356.

\section{Declarations}

Conflict of interest All authors are current or former members of the Bacterial and Archaeal Viruses Subcommittee, Bacterial Viruses Subcommittee and/or Archaeal Viruses Subcommittee of the International Committee on Taxonomy of Viruses (ICTV).

Open Access This article is licensed under a Creative Commons Attribution 4.0 International License, which permits use, sharing, adaptation, distribution and reproduction in any medium or format, as long as you give appropriate credit to the original author(s) and the source, provide a link to the Creative Commons licence, and indicate if changes were made. The images or other third party material in this article are included in the article's Creative Commons licence, unless indicated otherwise in a credit line to the material. If material is not included in the article's Creative Commons licence and your intended use is not permitted by statutory regulation or exceeds the permitted use, you will need to obtain permission directly from the copyright holder. To view a copy of this licence, visit http://creativecommons.org/licenses/by/4.0/. 


\section{References}

1. Gorbalenya AE, Krupovic M, Mushegian A et al (2020) The new scope of virus taxonomy: partitioning the virosphere into 15 hierarchical ranks. Nat Microbiol 5:668-674. https://doi.org/10.1038/ s41564-020-0709-x

2. Koonin EV, Dolja VV, Krupovic M et al (2020) Global organization and proposed megataxonomy of the virus world. Microbiol Mol Biol Rev 84:1-33. https://doi.org/10.1128/MMBR.00061-19

3. Walker PJ, Siddell SG, Lefkowitz EJ et al (2020) Changes to virus taxonomy and the Statutes ratified by the International Committee on Taxonomy of Viruses (2020). Arch Virol 165:2737-2748. https://doi.org/10.1007/s00705-020-04752-x

4. Baquero DP, Liu Y, Wang F et al (2020) Structure and assembly of archaeal viruses. Adv Virus Res 108:127-164

5. Krupovic M, Kuhn JH, Wang F et al (2021) Adnaviria: a new realm for archaeal filamentous viruses with linear A-form double-stranded DNA genomes. J Virol. https://doi.org/10.1128/JVI. 00673-21

6. Prangishvili D, Krupovic M (2012) A new proposed taxon for double-stranded DNA viruses, the order "Ligamenvirales." Arch Virol 157:791-795. https://doi.org/10.1007/s00705-012-1229-7

7. Wittmann J, Turner D, Millard A et al (2020) From orphan phage to a proposed new family-the diversity of N4-like viruses. Antibiotics 9:663. https://doi.org/10.3390/antibiotics9100663

8. Bischoff V, Bunk B, Meier-Kolthoff JP et al (2019) Cobavirusesa new globally distributed phage group infecting Rhodobacteraceae in marine ecosystems. ISME J 13:1404-1421. https://doi. org/10.1038/s41396-019-0362-7

9. Callanan J, Stockdale SR, Shkoporov A et al (2020) Expansion of known ssRNA phage genomes: from tens to over a thousand. Sci Adv 6:eaay5981. https://doi.org/10.1126/sciadv.aay5981

10. Kauffman KM, Hussain FA, Yang J et al (2018) A major lineage of non-tailed dsDNA viruses as unrecognized killers of marine bacteria. Nature 554:118-122. https://doi.org/10.1038/natur e25474

11. Pawlowski A, Rissanen I, Bamford JKH et al (2014) Gammasphaerolipovirus, a newly proposed bacteriophage genus, unifies viruses of halophilic archaea and thermophilic bacteria within the novel family Sphaerolipoviridae. Arch Virol 159:1541-1554. https://doi.org/10.1007/s00705-013-1970-6

12. Demina T, Pietilä M, Svirskaitè J et al (2017) HCIV-1 and other tailless icosahedral internal membrane-containing viruses of the family sphaerolipoviridae. Viruses 9:32. https://doi.org/10.3390/ v9020032

13. Roux S, Krupovic M, Daly RA et al (2019) Cryptic inoviruses revealed as pervasive in bacteria and archaea across Earth's biomes. Nat Microbiol 4:1895-1906. https://doi.org/10.1038/ s41564-019-0510-x

14. Barylski J, Kropinski AM, Alikhan N-F, Adriaenssens EM (2020) ICTV virus taxonomy profile: herelleviridae. J Gen Virol 101:3-4. https://doi.org/10.1099/jgv.0.001392

15. Prangishvili D, Mochizuki T, Krupovic M (2020) ICTV virus taxonomy profile: spiraviridae. J Gen Virol 101:240-241. https:// doi.org/10.1099/jgv.0.001385

16. Huang L, Wang H (2021) ICTV virus taxonomy profile: ovaliviridae. J Gen Virol. https://doi.org/10.1099/jgv.0.001546

17. Mäntynen S, Laanto E, Sundberg LR et al (2020) ICTV virus taxonomy profile: Finnlakeviridae. J Gen Virol 101:894-895. https:// doi.org/10.1099/jgv.0.001488

Publisher's Note Springer Nature remains neutral with regard to jurisdictional claims in published maps and institutional affiliations.

\title{
Authors and Affiliations
}

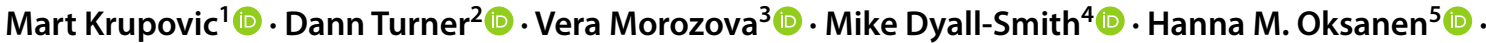

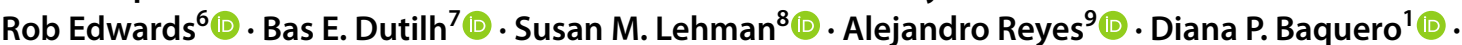

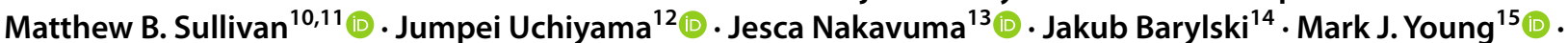

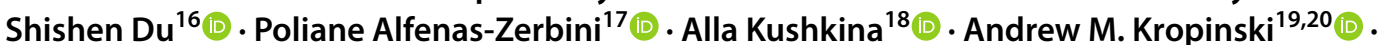

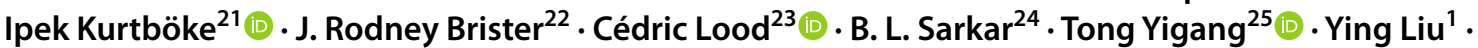

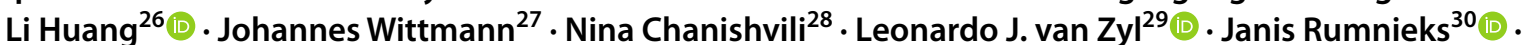 \\ Tomohiro Mochizuki ${ }^{31}$ (1) Matti Jalasvuori ${ }^{32}$ (1) Ramy K. Aziz ${ }^{33}$ (1) Małgorzata Łobocka ${ }^{34}$ (1)

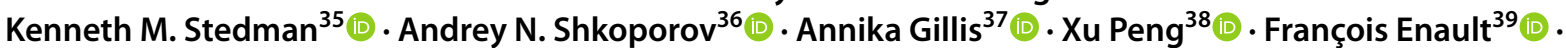

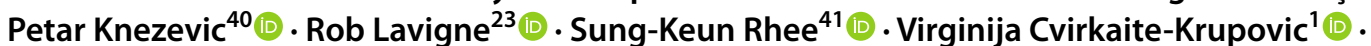 \\ Cristina Moraru ${ }^{42}$ - Andrea I. Moreno Switt ${ }^{43}$ - Minna M. Poranen ${ }^{5}$. Andrew Millard ${ }^{44}$ - David Prangishvili ${ }^{45}$. \\ Evelien M. Adriaenssens ${ }^{46}$ (i)
}

1 Archaeal Virology Unit, Institut Pasteur, Paris, France

2 Department of Applied Sciences, Faculty of Health and Applied Sciences, University of the West of England, Bristol, UK

3 Laboratory of Molecular Microbiology, Institute of Chemical Biology and Fundamental Medicine SB RAS, Novosibirsk, Russia

4 Veterinary Biosciences, Faculty of Veterinary and Agricultural Sciences, University of Melbourne, Parkville, Australia
Molecular and Integrative Biosciences Research Programme, Faculty of Biological and Environmental Sciences, University of Helsinki, Helsinki, Finland

6 Flinders Accelerator for Microbiome Exploration, Adelaide, Australia

7 Theoretical Biology and Bioinformatics, Department of Biology, Utrecht University, Utrecht, Netherlands

8 Center for Biologics Evaluation and Research, US Food and Drug Administration, 10903 New Hampshire Ave, Silver Spring, MD 20903, USA 
9 Max Planck Tandem Group in Computational Biology, Department of Biological Sciences, Universidad de Los Andes, Bogotá, Colombia

10 Department of Microbiology, The Ohio State University, Columbus, OH 43210, USA

11 Department Civil, Environmental, and Geodetic Engineering, The Ohio State University, Columbus, OH 43210, USA

12 School of Veterinary Medicine, Azabu University, Sagamihara, Japan

13 College of Veterinary Medicine, Animal Resources and Biosecurity, Makerere University, Kampala, Uganda

14 Department of Molecular Virology, Faculty of Biology, Institute of Experimental Biology, Adam Mickiewicz University in Poznan, Collegium Biologicum-Umultowska 89, 61-614 Poznan, Poland

15 Department of Plant Sciences and Plant Pathology, Montana State University, Bozeman, MT, USA

16 Department of Microbiology, College of Life Sciences, Wuhan University, Wuhan, China

17 Department of Microbiology, Universidade Federal de Viçosa, Viçosa, Brazil

18 Zabolotny Institute of Microbiology and Virology, NAS of Ukraine, Kyiv, Ukraine

19 Department of Food Science, University of Guelph, Guelph, Canada

20 Department of Pathobiology, University of Guelph, Guelph, Canada

21 University of the Sunshine Coast, Sippy Downs, Australia

22 National Center for Biotechnology Information, National Library of Medicine, National Institutes of Health, Bethesda, USA

23 KU Leuven, Leuven, Belgium

24 Emeritus ICMR-National Institute of Cholera and Enteric Diseases (NICED), Kolkata, India

25 Beijing University of Chemical Technology, Beijing, China

26 State Key Laboratory of Microbial Resources, Institute of Microbiology, Chinese Academy of Sciences, Beijing, China

27

Leibniz Institute DSMZ-German Collection of Microorganisms and Cell Cultures $\mathrm{GmbH}$, Berlin, Germany
28 The Eliava Institute of Bacteriophage, MIcrobiology and Virology, Tbilisi, Georgia

29 University of the Western Cape, Western Cape, South Africa

30 Latvian Biomedical Research and Study Center, Riga, Latvia

31 Earth-Life Science Institute, Tokyo Institute of Technology, Tokyo, Japan

32 University of Jyvaskyla, Jyvaskyla, Finland

33 Department of Microbiology and Immunology, Faculty of Pharmacy, Cairo University and Microbiology and Immunology Research Program, Children's Cancer Hospital Egypt, Cairo 57357, Egypt

34 Laboratory of Bacteriophage Biology, Institute of Biochemistry and Biophysics of the Polish Academy of Sciences, Warsaw, Poland

35 Biology Department and Center for Life in Extreme Environments, Portland State University, Portland, USA

36 University College Cork, Cork, Ireland

37 Laboratory of Food and Environmental Microbiology, Earth and Life Institute, UC Louvain, Louvain-la-Neuve, Belgium

38 Microbial Immunity Group, Department of Biology, University of Copenhagen, Copenhagen, Denmark

39 Université Clermont Auvergne, CNRS, LMGE, 63000 Clermont-Ferrand, France

40 Faculty of Sciences Department of Biology and Ecology, University of Novi Sad, Novi Sad, Serbia

41 Department of Biological Sciences and Biotechnology, Chungbuk National University, Cheongju, South Korea

42 Institute for Chemistry and Biology of the Marine Environment, University of Oldenburg, Oldenburg, Germany

43 Escuela de Medicina Veterinaria, Pontificia Universidad Católica de Chile, Santiago, Chile

44 Department of Genetics and Genome Biology, University of Leicester, Leicester, UK

45 Ivane Javakhishvili Tbilisi State University, Tbilisi, Georgia

46 Quadram Institute Biosciences, Norwich Research Park, Norwich NR4 7UQ, UK 